\title{
Alloying of Sn in the surface layer of $\operatorname{Ag}(111)$
}

\author{
Jacek Osiecki and Roger Uhrberg
}

\section{Linköping University Post Print}

N.B.: When citing this work, cite the original article.

Original Publication:

Jacek Osiecki and Roger Uhrberg, Alloying of $\mathrm{Sn}$ in the surface layer of $\operatorname{Ag}(111), 2013$, Physical Review B. Condensed Matter and Materials Physics, (87), 7.

http://dx.doi.org/10.1103/PhysRevB.87.075441

Copyright: American Physical Society http://www.aps.org/ 


\title{
Alloying of Sn in the surface layer of $\operatorname{Ag}(111)$
}

\author{
Jacek R. Osiecki and R. I. G. Uhrberg \\ Department of Physics, Chemistry, and Biology, Linköping University, S-581 83 Linköping, Sweden
}

(Received 22 November 2012; published 26 February 2013)

\begin{abstract}
It is found that $1 / 3$ monolayer (ML) of Sn forms a surface alloy with 2/3 ML of $\mathrm{Ag}$ on $\mathrm{Ag}(111)$. This highly ordered alloy layer shows a $\sqrt{3} \times \sqrt{3}$ structure. By employing experimental and theoretical tools (scanning tunneling microscopy [STM], angle resolved photoelectron spectroscopy, low-energy electron diffraction, and density functional theory), an atomic model has been obtained that reproduces the experimental electronic structure in both real and reciprocal space. Detailed surface band dispersions, constant energy contours, and STM images, obtained experimentally and theoretically, are compared in order to verify the model. Similar, 1-layer-thick alloys on $\mathrm{Ag}(111)$ with $\mathrm{Pb}, \mathrm{Bi}$, or $\mathrm{Sb}$ exhibit measurable spin-orbit interactions. However, no such spin split could be detected in the case of Sn in this study.
\end{abstract}

DOI: 10.1103/PhysRevB.87.075441 PACS number(s): 68.37.Ef, 79.60.Dp, 73.20.At, 71.15.Mb

\section{INTRODUCTION}

Alloying of elements at a surface, by an ordered replacement of atoms, leads to a two-dimensional (2D) periodic structure. Such an ordered surface alloy exhibits unique properties because of its confinement to a thin layer, which could be a single atomic layer thick. In this way, one can obtain an altered layer with a new 2D electronic structure. Interesting 2D alloy systems have been obtained on $\mathrm{Ag}(111)$, i.e., $\mathrm{Sb} / \mathrm{Ag}(111),{ }^{1} \mathrm{~Pb} / \mathrm{Ag}(111),{ }^{2}$ and $\mathrm{Bi} / \mathrm{Ag}(111) .{ }^{3}$ In all three cases, $\mathrm{Sb}, \mathrm{Pb}$, or $\mathrm{Bi}$ substitutes every third $\mathrm{Ag}$ atom of the surface layer, forming a $(\sqrt{3} \times \sqrt{3}) \mathrm{R} 30^{\circ}$ reconstruction. In this chemically changed layer, the electronic structure of the clean $\mathrm{Ag}(111)$ surface is modified as a result of hybridization of the electronic states of the surface species. The recent interest in surface alloys on $\mathrm{Ag}(111)$ is mainly driven by the Rashba type of spin split, ${ }^{1-6}$ observed for the surface bands, and its potential application related to spintronics. The Rashba split is large for $\mathrm{Bi} / \mathrm{Ag}(111)^{3}$ but barely detectable for $\mathrm{Sb} / \mathrm{Ag}(111){ }^{1}$ The trend of decreasing split with decreasing atomic number raises the question whether the Rashba split can be detected or not for the $\mathrm{Sn} / \mathrm{Ag}(111)$ surface alloy. However, the $\mathrm{Sn} / \mathrm{Ag}(111)$ surface alloy has not yet been reported in the literature, and an investigation of the atomic and electronic structure is therefore of interest on its own, as well as of interest as a complement to the $\mathrm{Bi}, \mathrm{Pb}$, and $\mathrm{Sb}$ cases. Our interest in the alloying properties of $\mathrm{Sn}$ and $\mathrm{Ag}$ was further triggered by the recent finding that these elements form well-ordered, 2D, surface alloys on both $\mathrm{Si}(111)$ and $\mathrm{Ge}(111))^{7,8}$

Here, we investigate the alloying of $1 / 3$ monolayer (ML) of $\mathrm{Sn}$ in the $\operatorname{Ag}(111)$ surface, resulting in a $\sqrt{3} \times \sqrt{3}$ periodicity. Scanning tunneling microscopy (STM) and angle resolved photoelectron spectroscopy (ARPES), were used to obtain atomic and electronic structure information. We compared the experimental results with those of density functional theory (DFT) calculations performed on a model, where Sn atoms substitute 1/3 ML of the Ag atoms in the surface layer. This is a model that has also been used to describe the alloying of $\mathrm{Sb}, \mathrm{Pb}$, and $\mathrm{Bi}$ with $\mathrm{Ag}(111)$. The calculations were done without introducing spin-orbit coupling, since no split is observed experimentally. We found good agreement between experiment and theory regarding both electronic band structure and STM images. The atomic and electronic structures of the
$\mathrm{Sn} / \mathrm{Ag}(111)$ surface alloy are similar to the $\mathrm{Sb}, \mathrm{Pb}$, and Bi cases mentioned previously except that no Rashba type of spin split could be resolved experimentally. In addition to the two major bands, which show various degrees of spin split as reported for the $\mathrm{Sb}, \mathrm{Pb}$, and $\mathrm{Bi}$ induced alloys, we identified a third weak dispersive feature not reported so far for this class of alloy on $\operatorname{Ag}(111)$. The origin of this feature is discussed in the paper.

\section{EXPERIMENTAL DETAILS}

STM experiments were performed in an ultra-high-vacuum system at Linköping University equipped with a variabletemperature STM (Omicron). ARPES experiments were performed using synchrotron radiation at beam line I4 at MAXlab, Sweden. The photoemission data were collected using a Phoibos 100 analyzer with a 2D detector (Specs). The total energy resolution of the ARPES data obtained at $27 \mathrm{eV}$ and presented in this paper is $\sim 40 \mathrm{meV}$, and the angular resolution is $0.3^{\circ}$.

To produce a clean and well-ordered $\mathrm{Ag}(111)$ surface, the single crystal was subjected to sputtering, using $1-\mathrm{keV}$ Ar ions at an Ar pressure of $5 \times 10^{-6}$ mbar followed by annealing at $400{ }^{\circ} \mathrm{C}$ for $2 \mathrm{~min}$. The number of sputtering cycles needed was judged from the quality of the low-energy electron diffraction (LEED) pattern and the intensity of the surface state in normal emission, i.e., the Shockley state. ${ }^{9}$ The preparation of the $\mathrm{Sn} / \mathrm{Ag}(111)-\sqrt{3} \times \sqrt{3}$ structure involved deposition of $0.36 \mathrm{ML}$ of $\mathrm{Sn}$, from a source calibrated using a quartz crystal monitor, onto the $\operatorname{Ag}(111)$ surface kept at $200^{\circ} \mathrm{C}$. The heating of the crystal was terminated immediately after the deposition. After this procedure, the structure of the surface had changed to a $\sqrt{3} \times \sqrt{3}$ periodicity as verified by the LEED pattern, which exhibited sharp diffraction spots as seen in Fig. 1(a).

\section{CALCULATIONAL METHODS}

Structural and electronic properties of a $\sqrt{3} \times \sqrt{3}$ atomic model of the 2D Sn/Ag surface alloy, with 1/3 ML of Sn in substitutional sites, were computed using DFT within the generalized gradient approximation, as implemented in the WIEN2k package. ${ }^{10}$ For this purpose, a slab was constructed, which possessed inversion symmetry; the surface layer, on each side of the slab, had a $\sqrt{3} \times \sqrt{3}$ structure with a 

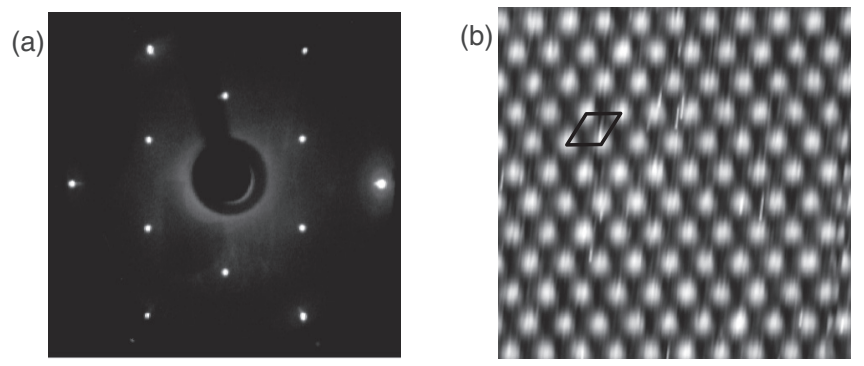

FIG. 1. (a) LEED pattern $(41 \mathrm{eV})$ exhibiting very sharp $\sqrt{3} \times \sqrt{3}$ spots, indicating a well-developed reconstruction. (b) Atomically resolved empty-state STM image of a $5 \times 5 \mathrm{~nm}^{2}$ area showing hexagonally arranged protrusions. The image was recorded in a constant current mode with a tunneling current of $200 \mathrm{pA}$ and a sample bias of $+0.3 \mathrm{~V}$. A $\sqrt{3} \times \sqrt{3}$ unit cell is drawn in the image.

2:1 Ag-to-Sn ratio. The slab superstructure had a lateral size corresponding to the $\sqrt{3} \times \sqrt{3}$ unit cell and a vertical dimension of $50 \AA$ that comprises 2 surface layers, containing both $\mathrm{Ag}$ and $\mathrm{Sn}$, and $12 \mathrm{Ag}$ layers between. Consecutive slabs were separated by a 13.2 - $\AA$-wide vacuum gap. The atomic model calculations were carried out with five $k$ points in the irreducible Brillouin zone and an energy cutoff of $81 \mathrm{eV}$. All atomic positions in the surface layer ( $\mathrm{Sn}$ and $\mathrm{Ag}$ atoms) and in the two Ag layers closest to the surface were optimized, and the force components for each atom were below $0.1 \mathrm{eV} / \AA$. Simulated STM images were generated from local density of states according to the Tersoff-Hamann approach. ${ }^{11}$

\section{EXPERIMENTAL RESULTS}

The $\operatorname{Sn} / \operatorname{Ag}(111)-\sqrt{3} \times \sqrt{3}$ surface was probed with the STM working in constant current mode at $200 \mathrm{pA}$. An atomically resolved, empty-state STM image of the $\sqrt{3} \times \sqrt{3}$ structure, collected at a sample bias of $+0.3 \mathrm{~V}$, is shown in Fig. 1(b). The image reveals atomic features, represented by round protrusions, which are arranged in a hexagonal pattern. The unit cell corresponds to a $\sqrt{3} \times \sqrt{3}$ periodicity, and it is outlined in the figure. Based on the deposited amount of $\mathrm{Sn}$, we assign the bright protrusions observed by STM to the positions of the Sn atoms.

The band structure of the valence electronic states of the $\mathrm{Sn} / \mathrm{Ag}(111)-\sqrt{3} \times \sqrt{3}$ surface was measured by ARPES. The investigated energy range had to be limited to $3.8 \mathrm{eV}$ below the Fermi level $\left(E_{F}\right)$ in order to avoid the very intense emission from the $\mathrm{Ag} 4 d$ bands, which would have saturated the detector, resulting in difficulties obtaining the surface band structure.

The Shockley surface state around $\bar{\Gamma}$, which is the signature of the clean $\operatorname{Ag}(111)$ surface, is no longer observed on the Sn alloyed one. Instead, the ARPES data along the two high-symmetry directions of the $\sqrt{3} \times \sqrt{3}$ surface Brillouin zone (SBZ), i.e., the $\bar{\Gamma} \bar{K}$ direction, shown in Fig. 2(a), and the $\bar{\Gamma} \bar{M}$ direction, shown in Fig. 2(b), revealed two well-resolved surface-state bands $S_{1}$ and $S_{2}$ that follow the periodicity of the $\sqrt{3} \times \sqrt{3}$ unit cell. In addition, a third weak dispersive feature labeled DF, which can only be observed at high emission angles, has been identified near $\bar{M}$ points of the $\sqrt{3} \times \sqrt{3} \mathrm{SBZ}$. There are also features related to Ag bulk emission in Figs. 2(a)
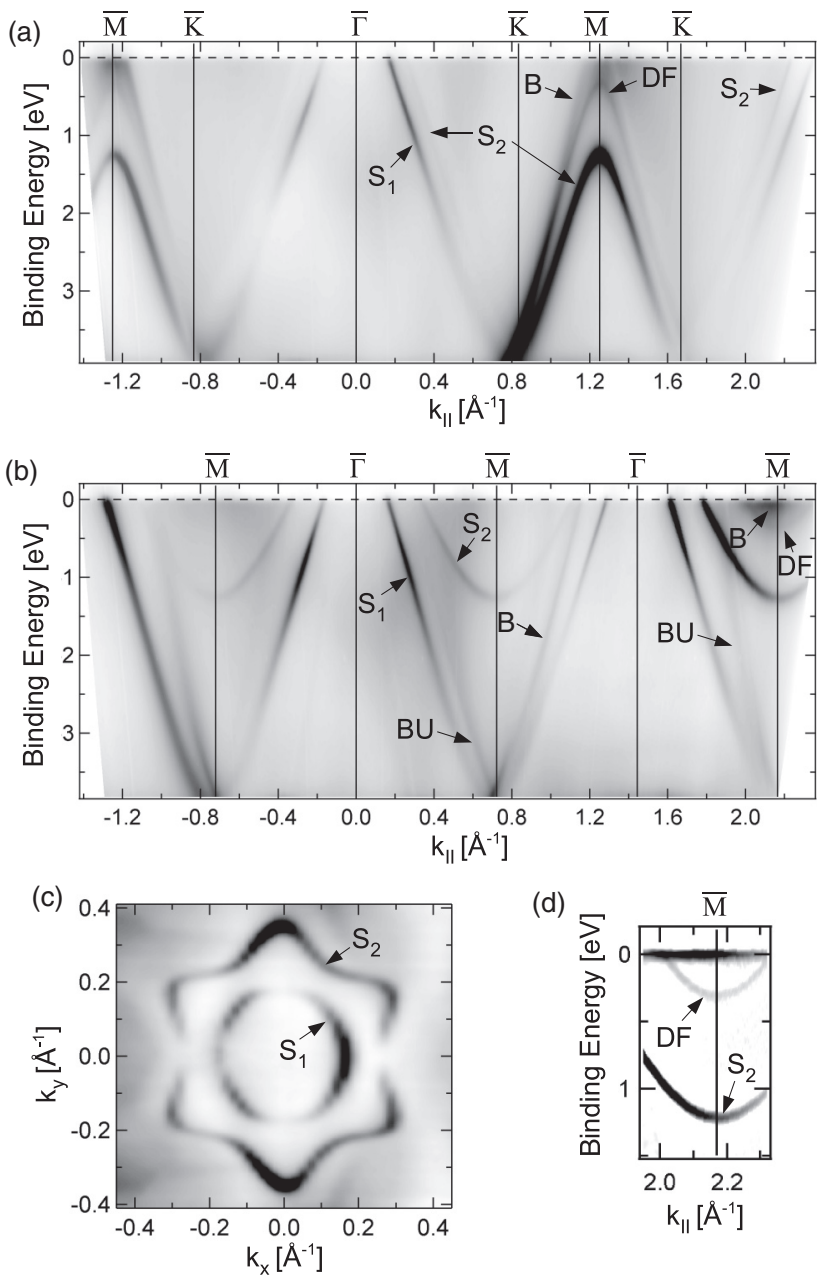

(d)

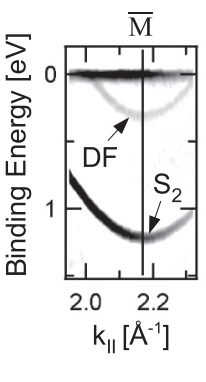

FIG. 2. Dispersion of the valence surface states, obtained using a photon energy of $27 \mathrm{eV}$, (a) along the $\bar{\Gamma} \bar{K}$ direction and (b) along the $\bar{\Gamma} \bar{M}$ direction. Two surface states $S_{1}$ and $S_{2}$, which show a $\sqrt{3} \times \sqrt{3}$ periodicity, are indicated by arrows. Both bands are unoccupied in the vicinity of the $\bar{\Gamma}$ point, and they disperse across the Fermi level, downward from the $\bar{\Gamma}$ point in the two high-symmetry directions. $S_{1}$ has a global minimum at the $\bar{M}$ point, and the $\bar{K}$ point is a global minimum of $S_{2}$. The $\bar{M}$ point is a saddle point of $S_{2}$. Other dispersive features are indicated by DF, B, and BU. DF is observed in the vicinity of the $\bar{M}$ point in (a) and the second $\bar{M}$ point in (b). Feature $\mathrm{B}$ is the silver bulk emission, and BU is an umklapp scattering of B. (c) Constant energy contours obtained at $0.1 \mathrm{eV}$ below the Fermi level. The inner hexagonlike contour corresponds to the $S_{1}$ band, and the outer contour with a flowerlike shape corresponds to the $S_{2}$ band. The $k_{x}$ axis is parallel to a $\bar{\Gamma} \bar{K}$ direction. The second derivative of the ARPES intensity around the second $\bar{M}$ point in (b) is shown in (d), revealing the dispersion of DF. The nondispersive line is the second derivative of the intensity drop at the Fermi level.

and 2(b), labeled B, and features due to umklapp scattering of the Ag bulk emission by the $\sqrt{3} \times \sqrt{3}$ structure, labeled BU. All bulk-related features that are observed in Figs. 2(a) and 2(b) have also been observed on $\mathrm{Pb} / \mathrm{Ag}(111)-\sqrt{3} \times \sqrt{3}^{2}$. The bands $S_{1}$ and $S_{2}$ are clearly visible as constant energy contours obtained at $0.1 \mathrm{eV}$ below $E_{F}$ in the proximity of the $\bar{\Gamma}$ point in Fig. 2(c). The inner, hexagonlike contour corresponds to the $S_{1}$ band, and the outer contour with a flowerlike shape 

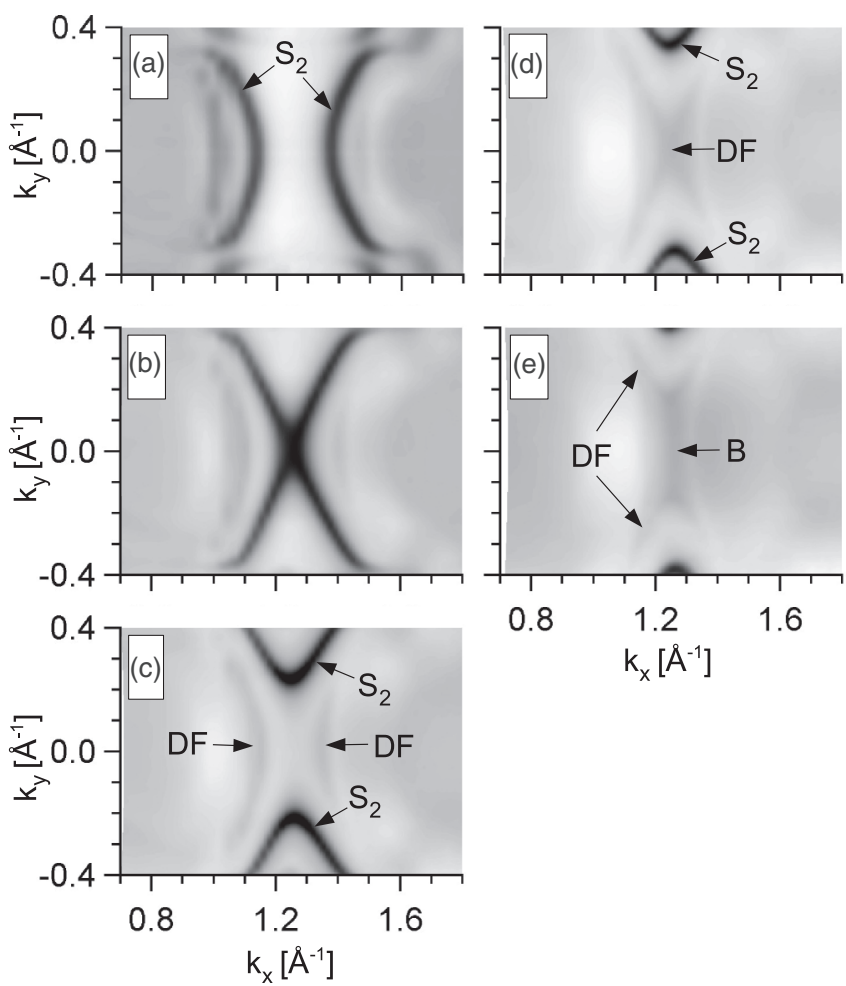

FIG. 3. Set of constant energy contours obtained at various binding energies, i.e., (a) at $1.8 \mathrm{eV}$, (b) at $1.21 \mathrm{eV}$, (c) at $0.8 \mathrm{eV}$, (d) at $0.31 \mathrm{eV}$, and (e) at $0.05 \mathrm{eV}$. The images are centered at the $\bar{M}$ point at $1.25 \AA^{-1}$ in Fig. 2(a), and the $k_{x}$ axis is parallel to the $\bar{K} \bar{M} \bar{K}$ direction, while the $k_{y}$ axis is parallel to the $\bar{\Gamma} \bar{M}$ direction. The contours display the momentum distribution of the $S_{2}$ and DF bands at certain binding energies. Both bands show similar contours, implying that the dispersions are qualitatively similar. The diffuse $\mathrm{Ag}$ emission is indicated by $\mathrm{B}$.

corresponds to the $S_{2}$ band. The emission intensity of $S_{2}$ is very weak between $\bar{\Gamma}$ and $\bar{K}$ in Fig. 2(a), which corresponds to the vanishing intensity of the $S_{2}$ contour at $k_{y}=0$ in Fig. 2(c). Both the $S_{1}$ and the $S_{2}$ bands are metallic, and in the vicinity of the $\bar{\Gamma}$ point they are unoccupied. The bands disperse downward from the $\bar{\Gamma}$ point and appear at the Fermi level at 0.18 and $0.38 \AA^{-1}$, respectively, as measured along $\bar{\Gamma} \bar{M}$ in Fig. 2(b). Except for a small region close to $\bar{M}$, the $S_{1}$ band is entirely contained within the measured energy range as in Fig. 2(b). Based on the symmetry with respect to the $\bar{M}$ point, we can conclude that $S_{1}$ has an energy minimum at this point, which is estimated to be $\sim 4.1 \mathrm{eV}$ below $E_{F}$. However, the occupied part of the $S_{2}$ band can be precisely outlined. Following the $\bar{\Gamma} \bar{K}$ direction in Fig. 2(a), we find that $S_{2}$ reaches a minimum at the $\bar{K}$ point, which is partly obscured due to the overlap of the Ag bulk emission in that region. The minimum of the $S_{2}$ band is $\sim 3.7 \mathrm{eV}$ below $E_{F}$ at the $\bar{K}$ point. From the $\bar{K}$ point until the $\bar{M}$ point, the $S_{2}$ band has an upward dispersion, and it has a binding energy of $1.21 \mathrm{eV}$ at the $\bar{M}$ point, as measured in Figs. 2(a) and 2(b). Following the $\bar{\Gamma} \bar{M}$ direction in Fig. 2(b), the $S_{2}$ band has a downward dispersion until the $\bar{M}$ point. The $\bar{M}$ point is thus a saddle point of the $S_{2}$ band. There is also a third weak dispersive feature, DF, which can be noticed in the vicinity of the $\bar{M}$ point at $2.17 \AA^{-1}$ along the $\bar{\Gamma} \bar{M}$ direction, where it forms a $0.31-\mathrm{eV}$-deep electron pocket at the symmetry point. DF is also observed at the $\bar{M}$ point along $\bar{\Gamma} \bar{K}$, where it shows a dispersion maximum. In the limited regions where DF is observed, it has a dispersion similar to $S_{2}$, but the binding energy is lower by $\sim 0.9 \mathrm{eV}$. The clean $\mathrm{Ag}(111)$ sample shows structureless emission from $E_{F}$ to a binding energy of $0.2-0.3 \mathrm{eV}$ at the $\bar{M}$ points where DF is observed. A remnant of that emission is the main reason DF cannot be clearly resolved in Figs. 2(a) and 2(b). The obscuring effect of the featureless $\mathrm{Ag}$ emission can be removed by taking the second derivative of the photoemission as displayed in Fig. 2(d) around the outer $\bar{M}$ point of Fig. 2(b). This procedure reveals the dispersion of the DF band. Further information about DF can be gained from a mapping of the band on the $\left(k_{x}, k_{y}\right)$ plane at various binding energies around the $\bar{M}$ point of Fig. 2(a). Such constant energy contours are displayed in Fig. 3 for a set of binding energies ranging from $1.8-0.05 \mathrm{eV}$. Figures 3 (a) -3 (c) show the contours of $S_{2}$ at binding energies below, at, and above the saddle point, respectively. In Fig. 3(c), there are two weak arcs that resemble those of $S_{2}$ in Fig. 3(a). These arcs are the constant energy contours of DF. From Figs. 3(c)-3(e), we find that the change with binding energy mimics that of $S_{2}$. The dispersion of DF obtained in the $\bar{\Gamma} \bar{M}$ direction from these data is the same as that shown in Fig. 2(d).

\section{THEORETICAL RESULTS AND DISCUSSION}

We constructed an atomic model of the $\sqrt{3} \times \sqrt{3}$ structure as an ordered alloyed layer with one Sn atom and two Ag atoms in the unit cell, as shown in Fig. 4(a). The modified surface layer can be viewed as the clean surface, where every third $\mathrm{Ag}$ atom is replaced by a $\mathrm{Sn}$ atom. The structure was subjected to energy minimization calculations, which resulted in optimal atomic positions. We found that the Sn atoms in this relaxed model are recessed slightly; i.e., they are located $0.04 \AA$ A below a plane going through the centers of the $\mathrm{Ag}$ atoms of the surface layer. The surface band structure calculated along the $\bar{\Gamma} \bar{K} \bar{M} \bar{\Gamma}$ path is shown in Fig. 4(b). In this figure, the size of a circle corresponds to the strength of the surface character summed over all orbitals at a particular $k_{\|}$value. There are three main surface bands, $\Sigma_{1}, \Sigma_{2}$, and $\Sigma_{3}$, in the occupied part of the band structure, with dispersions similar to the three experimental bands $S_{1}, S_{2}$, and DF. The bands are hybridized because $\Sigma_{1}$ has a mixed $\mathrm{Ag} s, d$ and $\mathrm{Sn} s$ character, $\Sigma_{2}$ has a $\operatorname{Ag} p, d$ and Sn $p$ character and $\Sigma_{3}$ has a $\operatorname{Ag} s, p$ and Sn $s, p$ character. Similar characters of the two main bands, $\Sigma_{1}$ and $\Sigma_{2}$, were obtained for $\mathrm{Pb} / \mathrm{Ag}(111){ }^{2}$

The calculated band structure reproduces the experimental result quite well but with noticeable differences. The closest match is at the Fermi level, where the experimental and theoretical positions of the electronic states in momentum space are almost the same. With increasing binding energy, there is an increasing deviation between the experimental and the theoretical band structure. Thus, the measured and the calculated bandwidths and the absolute positions of the electronic states do not match exactly, e.g., $S_{2}$ has a binding energy of $1.21 \mathrm{eV}$ at the $\bar{M}$ point compared to $1.08 \mathrm{eV}$ for $\Sigma_{2}$. A better match cannot be established by a rigid shift of the theoretical results because the bandwidths are not the same.

For an easy comparison to the $S_{1}$ and $S_{2}$ bands in momentum space, we calculated constant energy contours of the $\Sigma_{1}$ and 

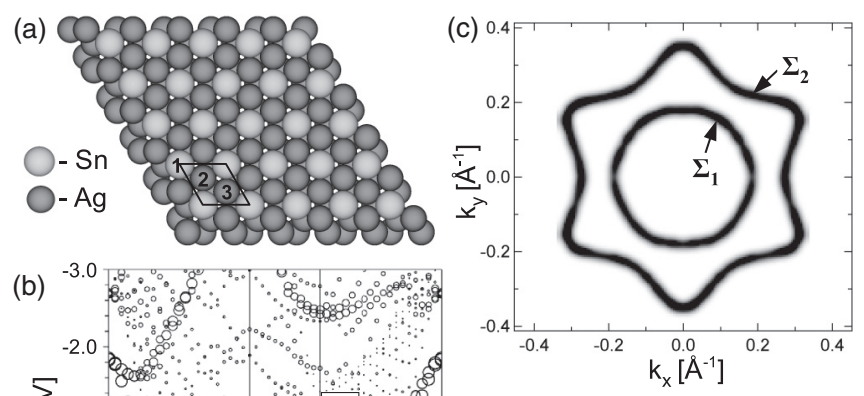

(b)

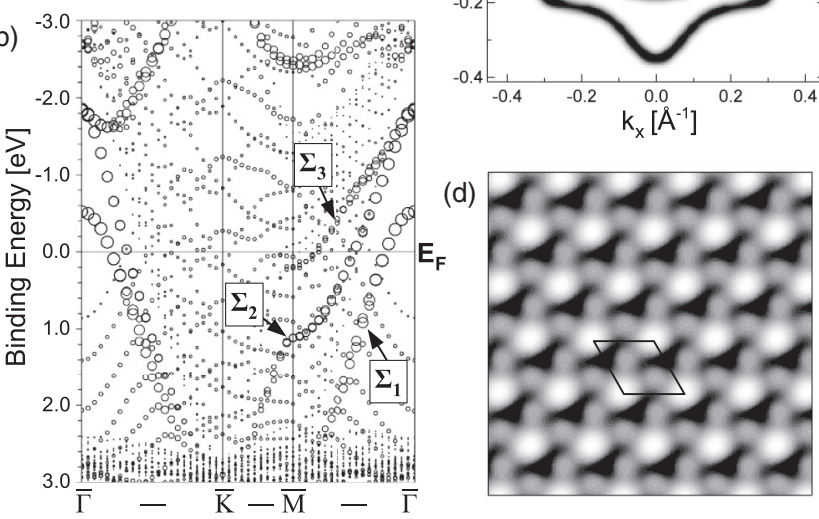

FIG. 4. (a) Atomic model of the $\sqrt{3} \times \sqrt{3}$ structure. The unit cell is outlined by the parallelogram. There is one Sn atom labeled 1 and two Ag atoms, labeled 2 and 3, in the unit cell. (b) Calculated electronic band structure of the model. Three metallic surface bands, $\Sigma_{1}, \Sigma_{2}$, and $\Sigma_{3}$, are present that reproduce the dispersion of the experimental bands $S_{1}, S_{2}$, and DF in Figs. 2(a), 2(b), and 2(d). The $S_{1}$ and $S_{2}$ bands are identified with the theoretical $\Sigma_{1}$ and $\Sigma_{2}$ bands, but the identification of DF is not straightforward, as discussed in the text. (c) Calculated constant energy contours, which are similar to the experimentally obtained ones in Fig. 2(c). (d) Simulated empty-state STM image of the model in (a). The image is calculated for the electronic states up to $0.3 \mathrm{eV}$ above the Fermi level. The $\sqrt{3} \times \sqrt{3}$ unit cell is outlined by parallelogram. The calculated STM image reproduces the appearance of the experimental STM image in Fig. 1(b).

$\Sigma_{2}$ bands at $0.1 \mathrm{eV}$ below $E_{F}$ in the proximity of the $\bar{\Gamma}$ point [Fig. 4(c)]. There are two contours: the inner one represents $\Sigma_{1}$, and the outer represents $\Sigma_{2}$. The shapes and the extensions of these curves in the momentum plane are identical to what is observed for the experimental bands $S_{1}$ and $S_{2}$ in Fig. 2(c). From this good agreement between experiment and theory found for $S_{1}-\Sigma_{1}$ and $S_{2}-\Sigma_{2}$, we conclude that the atomic structure of the $\mathrm{Sn} / \mathrm{Ag}(111)$ surface alloy is well described by the model in Fig. 4(a). This conclusion is further supported by results of band structure calculations that we performed on various models consistent with the $\sqrt{3} \times \sqrt{3}$ periodicity. These include models with 1/3 ML of Sn in adatom sites, 2/3 ML of Sn in substitutional sites, and a 2-layer-thick alloy with $1 / 3 \mathrm{ML}$ of $\mathrm{Sn}$ in each layer. All models gave surface band structures that were qualitatively different from the experimental band structure observed for the main surface bands $S_{1}$ and $S_{2}$.

The interpretation of DF is not as straightforward as the interpretation of the two main surface states. Comparing with the theoretical band structure, it seems that DF might be explained as emission from the $\Sigma_{3}$ band. The dispersions along $\bar{\Gamma} \bar{M}$ and the binding energies near the $\bar{M}$ points match. However, DF is only observable around the outer $\bar{M}$ points in Figs. 2(b) and 2(d). This could be understandable since the emission intensity is significantly lower inside the first Brillouin zone. Experimental data in Figs. 2(a) and 3 show that DF disperses downward from $\bar{M}$ to $\bar{K}$. Unfortunately, no comparison can be made with the theoretical band structure since $\Sigma_{3}$ cannot be identified between $\bar{M}$ and $\bar{K}$. A definite identification of DF with $\Sigma_{3}$ is therefore not possible. Furthermore, the emission associated with DF is very close to the bulk emission B from the $\operatorname{Ag}(111)$ crystal, which is discussed in Refs. 12 and 13 . Along $\bar{K} \bar{M} \bar{K}$, the DF emission appears to be split off from the $\mathrm{B}$ emission near $\bar{M}$. A similar impression is conveyed by Fig. 2(b), where the DF emission could be viewed as being split off from the diffuse $\mathrm{Ag}$ emission. Thus, an interpretation of DF as due to modified $\mathrm{Ag}$ bulk emission is an explanation that is a plausible alternative to the identification with the $\Sigma_{3}$ surface state.

A simulated empty-state STM image, which has been derived from calculated electronic states in the energy range 0 to $0.3 \mathrm{eV}$ above $E_{F}$, is shown in Fig. 4(d). The $\sqrt{3} \times \sqrt{3}$ unit cell is outlined by the parallelogram and the corners of it match the positions of the Sn atoms in the atomic model. Thus, the bright protrusions in the simulated STM image, forming the hexagonal pattern represent $\mathrm{Sn}$ atoms of the model in Fig. 4(a). This is consistent with the experimental STM image, collected with a sample bias of $+0.3 \mathrm{~V}$, shown in Fig. 1(b), where the bright protrusions were interpreted as Sn atoms, based on the deposited amount.

\section{SUMMARY}

Using experimental and theoretical tools, the atomic and electronic structures of the $\mathrm{Sn} / \operatorname{Ag}(111)-\sqrt{3} \times \sqrt{3}$ surface alloy were determined. The calculated electronic structure of a model where 1/3 ML of Sn occupies substitutional surface sites forming an ordered 2D structure is in good agreement with the ARPES results. The Rashba type of spin split of the surface bands, observed in the case of the $\mathrm{Sb}, \mathrm{Pb}$, and $\mathrm{Bi}$ surface alloys, could not be detected in the case of Sn. This observation is consistent with the decrease in the Rashba split with atomic number and with the split observed for Sb being already very close to the detection limit.

\section{ACKNOWLEDGMENTS}

Technical support from Dr. P. E. J. Eriksson at Linköping University, as well as from Dr. Johan Adell and Dr. T. Balasubramanian at MAX-lab, is gratefully acknowledged. Financial support was provided by the Swedish Research Council (Contract No. 621-2010-3746), the Linköping Linnaeus Initiative for Novel Functional Materials supported by the Swedish Research Council (Contract No. 2008-6582), and the Knut and Alice Wallenberg Foundation.
${ }^{1}$ L. Moreschini, A. Bendounan, I. Gierz, C. R. Ast, H. Mirhosseini, H. Höchst, K. Kern, J. Henk, A. Ernst, S. Ostanin, F. Reinert, and M. Grionial, Phys. Rev. B 79, 075424 (2009).
${ }^{2}$ D. Pacilé, C. R. Ast, M. Papagno, C. Da Silva, L. Moreschini, M. Falub, A. P. Seitsonen, and M. Grioni, Phys. Rev. B 73, 245429 (2006). 
${ }^{3}$ C. R. Ast, J. Henk, A. Ernst, L. Moreschini, M. C. Falub, D. Pacilé, P. Bruno, K. Kern, and M. Grioni, Phys. Rev. Lett. 98, 186807 (2007).

${ }^{4}$ L. Moreschini, A. Bendounan, H. Bentmann, M. Assig, K. Kern, F. Reinert, J. Henk, C. R. Ast, and M. Grioni, Phys. Rev. B 80, 035438 (2009).

${ }^{5}$ K. Sakamoto, T. Oda, A. Kimura, K. Miyamoto, M. Tsujikawa, A. Imai, N. Ueno, H. Namatame, M. Taniguchi, P. E. J. Eriksson, and R. I. G. Uhrberg, Phys. Rev. Lett. 102, 096805 (2009).

${ }^{6}$ Y. A. Bychkov and E. I. Rashba, JETP Lett. 39, 78 (1984).

${ }^{7}$ J. R. Osiecki, H. M. Sohail, P. E. J. Eriksson, and R. I. G. Uhrberg, Phys. Rev. Lett. 109, 057601 (2012).
${ }^{8}$ H. M. Sohail, J. R. Osiecki, and R. I. G. Uhrberg, Phys. Rev. B 85, 205409 (2012)

${ }^{9}$ F. Reinert, G. Nicolay, S. Schmidt, D. Ehm, and S. Hüfner, Phys. Rev. B 63, 115415 (2001).

${ }^{10}$ P. Blaha, K. Schwarz, G. K. H. Madsen, D. Kvasnicka, and J. Luitz, WIEN2K: An Augmented Plane Wave + Local Orbitals Program for Calculating Crystal Properties (Karlheinz Schwarz, Technische Universität, Vienna, Austria, 2001).

${ }^{11}$ J. Tersoff and D. R. Hamann, Phys. Rev. B 31, 805 (1985).

${ }^{12}$ A. Arranz, J. F. Sánchez-Royo, J. Avila, V. Pérez-Dieste, P. Dumas, and M. C. Asensio, Phys. Rev. B 65, 075405 (2002).

${ }^{13}$ J. F. Sánchez-Royo, J. Avila, V. Pérez-Dieste, M. De Seta, and M. C. Asensio, Phys. Rev. B 66, 035401 (2002). 